\title{
GESTACIÓN GEMELAR MONOCORIAL CON RCIU SELECTIVO TIPO 2: HALLAZGOS ECOGRÁFICOS Y FETOSCÓPICOS DE MAL PRONÓSTICO
}

\author{
Enrique Gil Guevara1', Sarah Bower², Kypros Nicolaides ${ }^{3}$
}

\begin{abstract}
RESUMEN
Presentamos el caso de una gestante de 17 semanas con una gestación gemelar monocoriónica biamniótica complicada con RCIU selectivo tipo 2 que requirió, de acuerdo a criterios ecográficos, ablación quirúrgica Láser de las anastomosis placentarias. Se identificó durante la fetoscopía una anastomosis arterio-venosa que mostraba flujo bidireccional lo cual es sumamente inusual en estas anastomosis, no reportado antes en la literatura, constituyendo un signo de mal pronóstico para el gemelo pequeño.
\end{abstract}

Palabras clave: Gestación gemelar monocorial; RCIU selectivo; Anastomosis arterio-venosa - Fetoscopía(Fuente: DeCS BIREME).

\section{MONOCHORIONIC DIAMNIOTIC TWIN PREGNANCY WITH SELECTIVE IUGR TYPE 2: SONOGRAPHIC AND FETOSCOPIC FINDINGS OF OMINOUS PROGNOSIS}

\begin{abstract}
A pregnant woman with monochorionic twins was referred to our Centre due to discrepancy in size between the twins. She was 17 weeks pregnant when we made the diagnosis of selective IUGR type 2 and we decided to perform a fetoscopic Laser ablation of the placental anastomoses. During the fetoscopy, we identified an arterio-venous anastomosis with bidirectional flux that is completely atypical in these anastomoses, never reported before, thus representing a sign of bad prognosis for the small twin.
\end{abstract}

Keywords: Monochorionic twin pregnancy; Selective IUGR; Arterio-venous anastomosis-Fetoscopy(Source:MeSH NLM).

\section{INTRODUCCIÓN}

Las gestaciones monocoriales presentan un gran reto diagnóstico y terapéutico debido a las distintas complicaciones que surgen del hecho de que ambos gemelos compartan la misma placenta.

Entre estas complicaciones tenemos el Síndrome de Transfusión Feto-Fetal, la Restricción Selectiva del Crecimiento IntraUterino, la Secuencia AnemiaPolicitemia Gemelar, la Secuencia de Perfusión Arterial Reversa Gemelar y la gestación monoamniótica.

Un punto importante a resaltar es que, debido a la circulación placentaria compartida por ambos gemelos, el bienestar de uno de ellos depende críticamente del bienestar del otro. De hecho, después de diagnosticar la muerte espontánea de uno de los gemelos, el sobreviviente presenta un riesgo de muerte de $15 \%$ y un riesgo de afectación neurológica del $25 \%$ debido a exanguinación aguda hacia el gemelo muerto a través de las anastomosis placentarias ${ }^{1}$.

Precisamente este último punto es sumamente importante y asimismo controversial en el manejo de una de las complicaciones propias de la monocorionicidad: la Restricción Selectiva del Crecimiento Intrauterino.

Comunicamos el caso de una gestación doble monocorial complicadacon RCIU selectivo, manejada quirúrgicamente, en la que describimos criterios ecográficos de severidad y, por primera vez en la literatura, un signo fetoscópico de mal pronóstico para el gemelo pequeño: una anastomosis arterio-venosa con flujo cambiante.

\section{CASO CLÍNICO}

Una primigesta de 23 años con una embarazo espontáneo gemelar monocoriónico diamniótico fue referida a nuestro centro a las 16 semanas de gestación. El motivo fue una marcada discrepancia en el crecimiento de ambos gemelos (Figura 1) y dopplers anormales en el gemelo pequeño.

La paciente contaba con un tamizaje de bajo riesgo para cromosomopatías en la ecografía del primer trimestre. No había una diferencia importante en las traslucencias nucales $(1.7 \mathrm{~mm}$ vs $1.9 \mathrm{~mm})$ pero la discrepancia en las longitudes corono-nalga era de $21 \%(63.4 \mathrm{~mm}$ vs $80.4 \mathrm{~mm})$.

\footnotetext{
Research Fellow en Medicina Fetal, King's College Hospital, Londres, Inglaterra

Consultant en Medicina Fetal, King's College Hospital, Londres, Inglaterra

Professor en Medicina Fetal, King's College Hospital, Londres, Inglaterra
} 


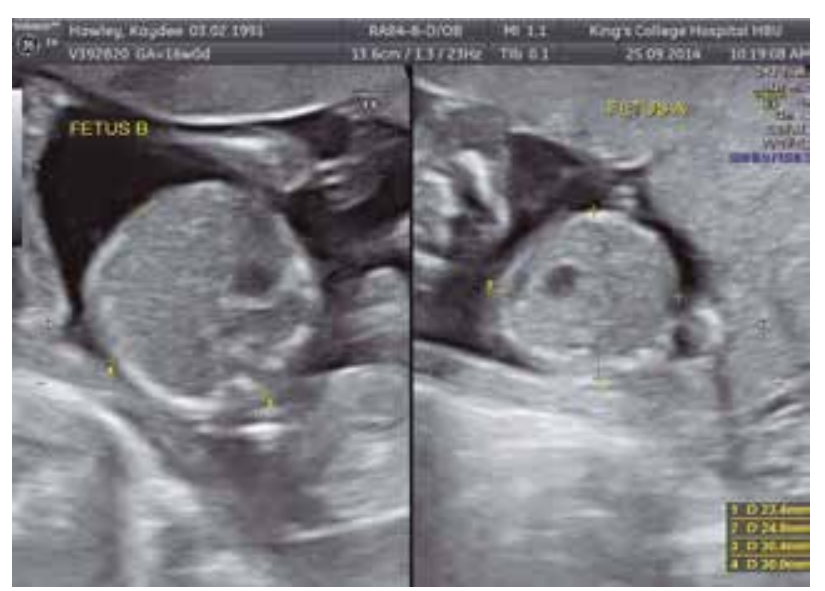

Figura 1. Discrepancia en los perímetros abdominales de ambos gemelos.

La paciente no era fumadora, su nivel de hemoglobina era normal y no sufría de ninguna enfermedad importante.

Realizamos la exploración ecográfica de esta paciente a las 16 semanas de gestación y encontramos una discrepancia del $45 \%$ en el peso de ambos gemelos con el perímetro abdominal del gemelo pequeño por debajo del percentil 5. La anatomía de ambos gemelos era normal.

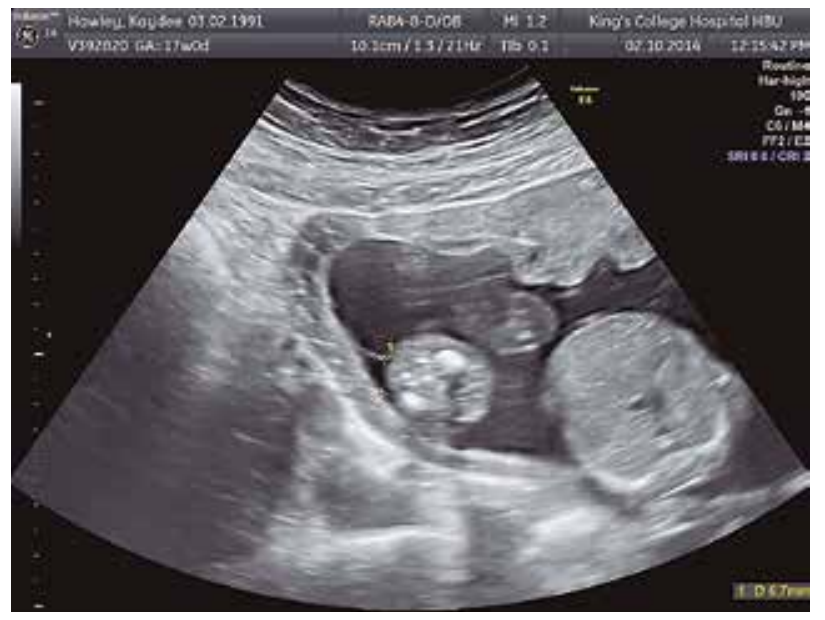

Figura 2. Oligoamnios en el gemelo pequeño.

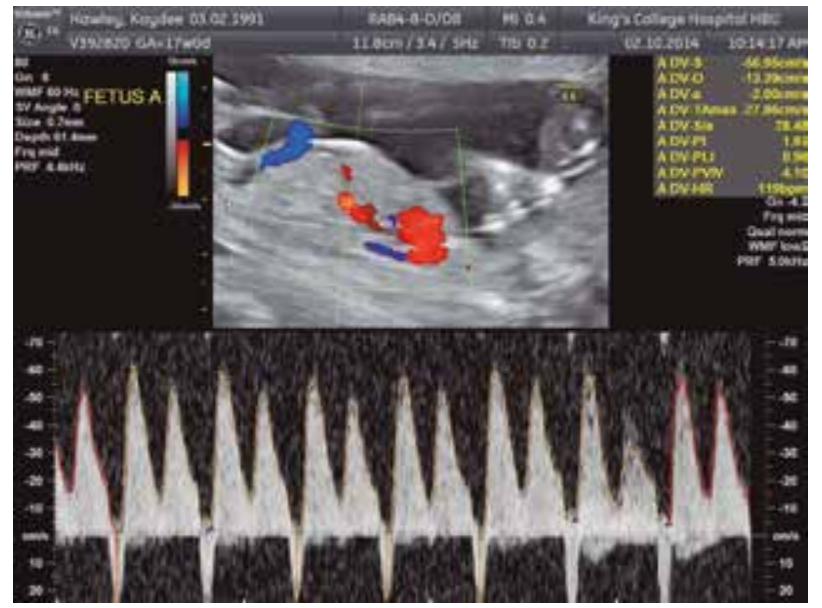

La cantidad de líquido amniótico en el gemelo pequeño estaba en el límite inferior normal y en el gemelo sano era normal.

El gemelo pequeño mostraba flujo diastólico ausente en una de las arterias umbilicales, flujo normal en la arteria cerebral media y un índice de pulsatilidad elevado en el ductus venoso pero con onda "a" positiva. Los dopplers en el gemelo grande eran normales.

Concluimos que se trataba de una gestación monocoriónica diamniótica con RCIU selectivo tipo 2 y decidimos controlarla en una semana con la probabilidad de cirugía láser en caso de deterioración del gemelo pequeño.

A la semana siguiente, ya con 17 semanas de gestación, la discrepancia se mantuvo en $45 \%$. El gemelo pequeño, aún por debajo del percentil 5, presentaba ahora oligoamnios con un pozo vertical máximo de $1 \mathrm{~cm}$ (Figura 2).

En cuanto a los dopplers, el gemelo pequeño mostraba ahora flujo diastólico ausente en ambas arterias umbilicales, onda "a" reversa en el ductus venoso y pico sistólico elevado en la arteria cerebral media (Figuras 3 y 4 ).

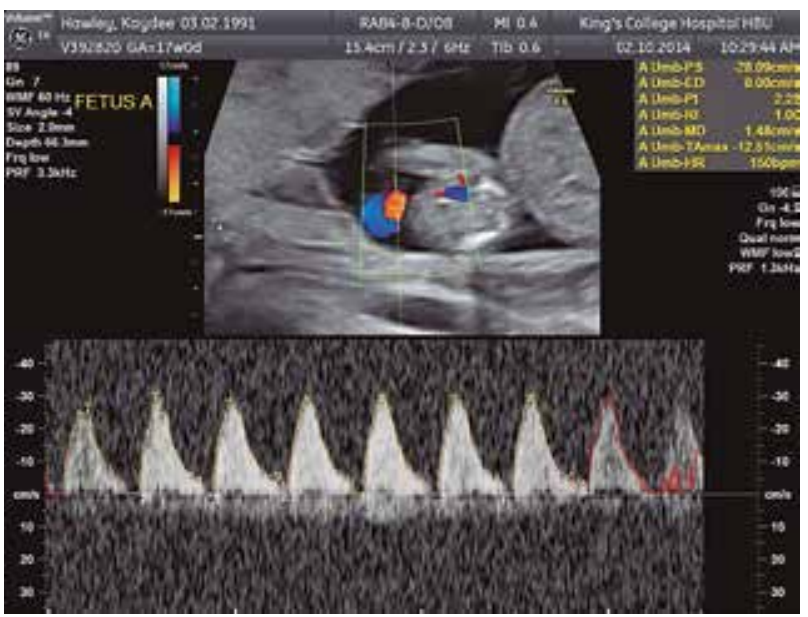

Figura 3. EDF ausente en la arteria umbilical.

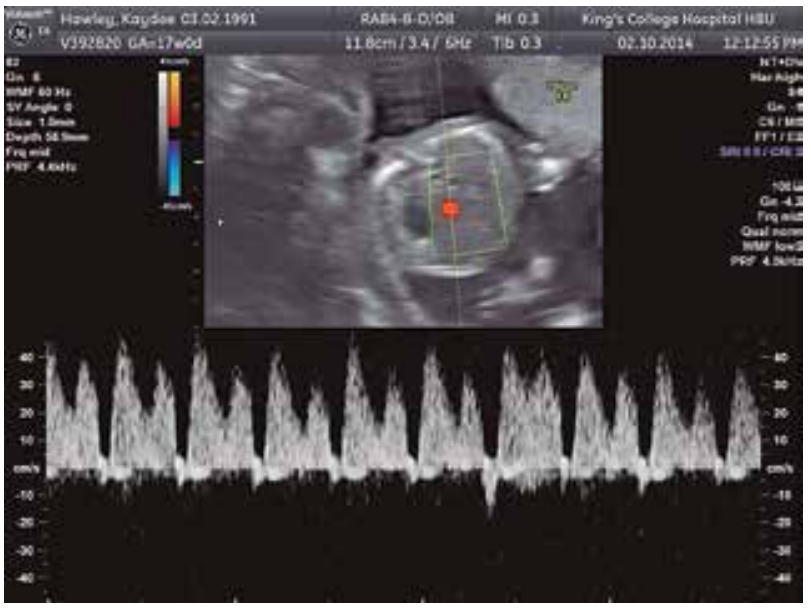

Figura 4. Onda "a" reversa en el ductus venoso del gemelo pequeño (Cortes sagital y axial). 


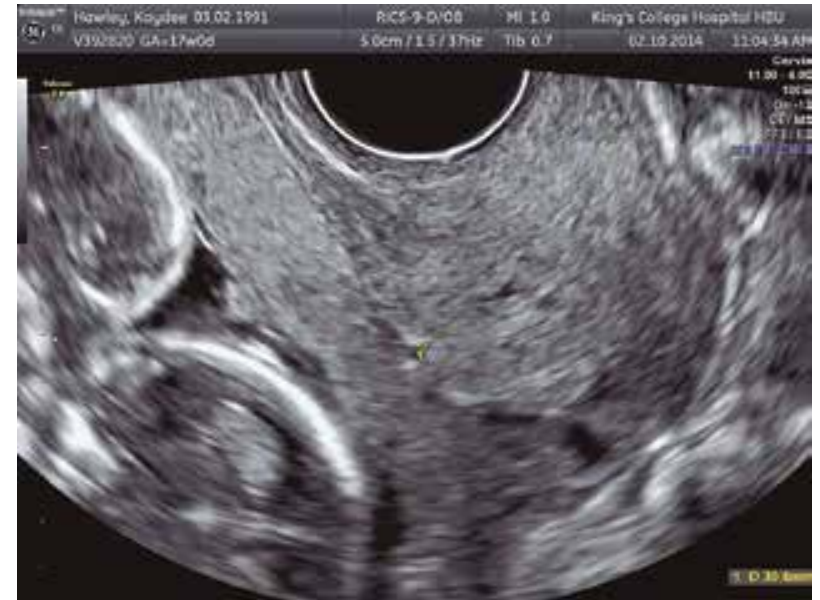

Figura 5. Medición del cérvix uterino.

El gemelo sano presentaba dopplers normales. El cérvix era largo, sin tunelización y medía $30 \mathrm{~mm}$ (Figura 5 ).

El diagnóstico era consistente: gestación gemelar monocoriónica diamniótica con RCIU selectivo tipo 2 . En vista de la deterioración del gemelo pequeño y de su alta probabilidad de muerte, decidimos realizar la cirugía principalmente para proteger el bienestar del gemelo sano en caso de muerte del gemelo enfermo.

Explicamos la gravedad de la situación a la paciente dejando claro que si el manejo era expectante, ambos fetos tendrían un alto riesgo de muerte o de daño cerebral. Con respecto al manejo quirúrgico, debido a la condición del gemelo pequeño, la chance de supervivencia de éste era sólo de $10 \%$ mientras que para el gemelo sano era de $70 \%{ }^{2}$.

La paciente aceptó el manejo quirúrgico y firmó el consentimiento informado.

La cirugía fetoscópica se realizó exitosamente, con anestesia local, y sin ninguna complicación materna. Asimismo, se obtuvo una muestra de líquido amniótico para análisis de cariotipo fetal, el cual resultó siendo normal.

Durante la fetoscopía, se realizó la ablación placentaria secuencial, identificando primero selectivamente las anastomosis y luego quemando a lo largo del ecuador placentario. Entre las anastomosis encontradas, se identificó claramente una anastomosis arterio-venosa que iba del territorio placentario del gemelo pequeño hacia el del gemelo sano y que presentaba un color fluctuante entre rojo oscuro y rojo vivo lo cual es sumamente inusual en este tipo de anastomosis (unidireccionales). Se consideró que esto reflejaba la baja presión vascular del gemelo pequeño que causaba que la presión en sus ramas arteriales placentarias sea vencida por la presión de las ramas venosas placentarias del gemelo sano. Por

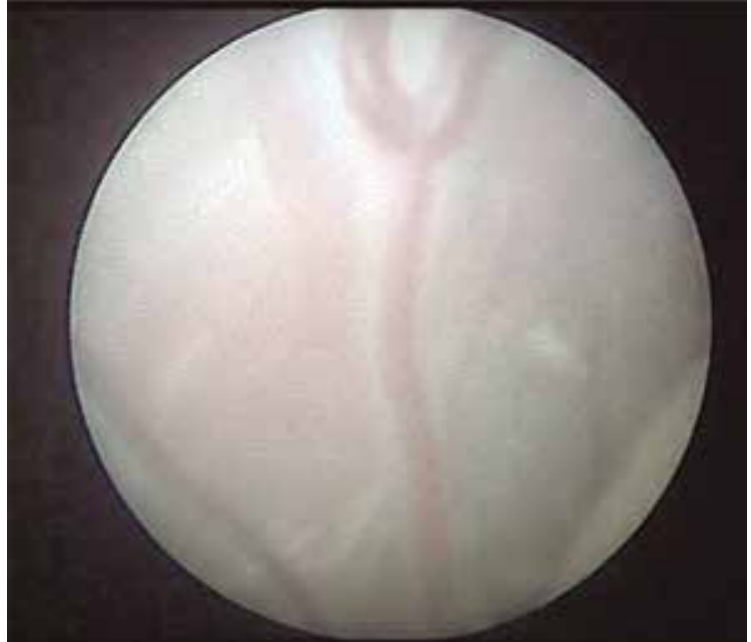

Figura 6. Anastomosis arterio-venosa del gemelo pequeño al gemelo sano.

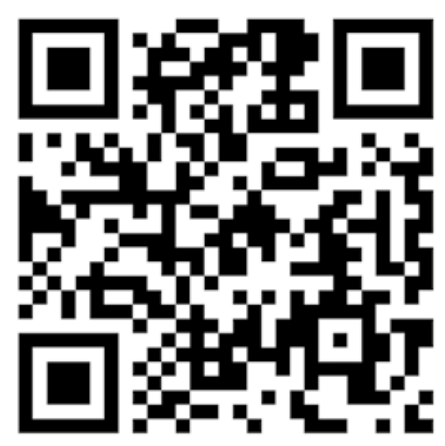

Escanee esta imagen con su Smartphone para ver el Video.

ende se procedió a quemar esta anastomosis en aras de proteger al gemelo normal (Figura 6).

La paciente fue dada de alta el mismo día. Una semana después la paciente presentó rotura prematura de membranas y en un control ecográfico cuatro semanas después de la cirugía, a las 21 semanas de gestación, se constató ausencia de actividad cardíaca en el gemelo pequeño.

La evolución del gemelo sano a lo largo del embarazo fue favorable. Nació a las 34 semanas 4 días, por cesárea electiva debido a oligoamnios, RPM prolongado y presentación podálica, con un peso de 2327 gramos y sin ninguna complicación. Su evolución psicomotriz, con 6 meses de edad, es normal a la fecha.

\section{DISCUSIÓN}

Las gestaciones monocoriales (que constituyen el $20 \%$ de las gestaciones dobles) presentan un riesgo de pérdida 
antes de las 24 semanas de $12 \%$, el cual es 6 veces mayor que el de las gestaciones dicoriales ${ }^{1}$.

Como mencionamos anteriormente, la circulación placentaria compartida por ambos gemelos genera complicaciones entre las cuales el RCIU selectivo se presenta en el $12 \%$ de las gestaciones monocoriales ${ }^{2}$.

Los criterios ecográficos para su diagnóstico son una discrepancia en tamaño igual o mayor al $25 \%$ y en el gemelo pequeño, antes de las 22 semanas, una circunferencia abdominal igual o menor al percentil 5 ó, con una edad gestacional igual o mayor a las 22 semanas, un peso fetal estimado igual o menor al percentil 5 .

Fisiopatológicamente, el RCIU selectivo se debe a la distribución inequitativa de la masa placentaria entre ambos gemelos. Es así que las anastomosis placentarias juegan un papel importante en su desarrollo ${ }^{3}$.

Las anastomosis arterio-venosas son unidireccionales y están presentes en el $95 \%$ de las gestaciones monocoriales, mientras que las anastomosis arterioarteriales son bidireccionales y esta propiedad las hace "protectoras" ya que ante cualquier desbalance entre los gemelos, el flujo sanguíneo es redirigido hacia el gemelo con menor oxigenación. El número de este tipo de anastomosis se asocia con el tipo específico de RCIU selectivo ${ }^{1,4}$.

Ecográficamente, el RCIU selectivo puede ser anticipado desde las 11-14 semanas al identificar una discrepancia considerable entre las longitudes corono-nalga de ambos gemelos como en el caso presentado ${ }^{2}$.

Una vez hecho el diagnóstico ecográfico, es importante clasificar el RCIU selectivo en tres tipos distintos. Esto se logra analizando ecográficamente el flujo doppler en la arteria umbilical del gemelo pequeño. El RCIU selectivo es tipo 1 cuando el flujo doppler es normal; tipo 2 cuando el flujo diastólico final es ausente o reverso; y tipo 3 cuando el flujo diastólico final es intermitentemente positivo-ausente-reverso ${ }^{5}$.

Cada uno de estos tipos tiene características placentarias, manejo y pronóstico distintos ${ }^{1}$.

EI RCIU selectivo tipo 2 muestra sólo en el 18\% de los casos anastomosis arterio-arteriales. Su manejo es por lo general quirúrgico ya que de no hacer nada en el $90 \%$ de los casos el gemelo pequeño muestra signos de deterioración y de muerte inminente ${ }^{6}$.

Los hallazgos ecográficos que ensombrecen el pronóstico de una gestación complicada con RCIU selectivo son la discrepancia de peso entre ambos gemelos (a mayor discrepancia peor pronóstico), el doppler de la arteria umbilical (flujo diastólico ausente o reverso) y del ductus venoso (onda "a" ausente o reversa) en el gemelo pequeño, la presencia de oligoamnios en éste, la edad gestacional al momento de la cirugía (a menor edad gestacional peor pronóstico) y finalmente la longitud del cérvix (a menor longitud cervical mayor riesgo de aborto o parto pretérmino) $)^{7}$.

Creemos importante comunicar este caso porque aparte de los hallazgos ecográficos identificados como signos de mal pronóstico, encontramos por primera vez un signo fetoscópico, no reportado anteriormente, que refleja el mal estado general del gemelo pequeño: una anastomosis arterio-venosa con flujo bidireccional propio de las anastomosis arterio-arteriales ${ }^{6}$ (Video Online).

La explicación de este fenómeno radica en la baja presión vascular de las ramas placentarias de las arterias umbilicales del gemelo pequeño que no son capaces de vencer la presión de las ramas placentarias de la vena umbilical del gemelo sano cuando aquéllas se mezclan con éstas en una anastomosis arterio-venosa. Esto produce fetoscópicamente un cambio de color constante (entre rojo oscuro y rojo claro) en la porción arterial de la anastomosis A-V (correspondiente al gemelo pequeño) y que, por ende, provoca un menor flujo sanguíneo de sangre venosa umbilical (oxigenada) hacia el gemelo sano por pérdida de ésta hacia el gemelo enfermo a través de dicha anastomosis A-V. Por esta razón, nos vimos en la necesidad de interrumpir esta anastomosis para proteger el flujo sanguíneo del gemelo sano y así evitar cualquier riesgo de daño cerebral en éste.

Finalmente, consideramos determinante, en el manejo de la restricción selectiva del crecimiento en gestaciones monocoriales, dos conductas:

Primero, una buena ecografía del primer trimestre que nos permita establecer claramente la corionicidad y amnionicidad de la gestación, así como las longitudes corono-nalga de ambos gemelos. $Y$ segundo, hacer una buena correlación ecográfico-fetoscópica que nos permita evaluar la progresión de la enfermedad y decidir el momento de la intervención así como enlazar los hallazgos ecográficos con los signos fetoscópicos aún por estudiar.

\section{REFERENCIAS BIBLIOGRÁFICAS}

1. Liesbeth Lewi; Jan Deprest; Kurt Hecher. The vascular anastomoses in monochorionic twin pregnancies and their clinical consequences. American Journal of Obstetrics \& Gynecology, Volume 208, Issue 1, 19-30. DOI: 10.1016/j. ajog.2012.09.025

2. Peeva G, Bower S, Orosz L, Chaveeva P, Akolekar R, Nicolaides K.H. Endoscopic Placental Laser Coagulation in Monochorionic Diamniotic Twins with Type II Selective Fetal Growth Restriction. Fetal Diagn Ther 2015; 38:86-93. DOI: 10.1159/000374109

3. Denbow, Mark L. et al. Placental angioarchitecture in monochorionic twin pregnancies: Relationship to fetal growth, fetofetal transfusion syndrome, and pregnancy outcome. American Journal of Obstetrics \& Gynecology, Vol 182, 
Issue 2, 417-426. DOI: http://dx.doi.org/10.1016/S00029378(00)70233-X

4. Valsky DV, Eixarch E, Martinez JM, Gratacós E. Selective intrauterine growth restriction in monochorionic diamniotic twin pregnancies. Prenat Diagn 2010; 30: 719-726. DOI: $10.1002 / p d .2536$

5. Valsky DV, Eixarch E, Martinez JM, Crispi F, Gratacós E. Selective intrauterine growth restriction in monochorionic twins: pathophysiology, diagnostic approach and management dilemmas. Semin Fetal Neonatal Med. 2010 Dec; 15(6): 3428. DOI: 10.1016/j.siny.2010.07.002

6. Lewi L, Cannie M, Blickstein I, et al. Placental sharing, birthweight discordance, and vascular anastomoses in monochorionic diamniotic twin placentas. Am J Obstet
Gynecol 2007; 197:587. e1-587.e8. DOI: 10.1016/j. ajog.2007.05.009

7. Ishii, K., Murakoshi, T., Hayashi, S., Saito, M., Sago, H., Takahashi, Y., Sumie, M., Nakata, M., Matsushita, M., Shinno, T., Naruse, H. and Torii, Y. (2011), Ultrasound predictors of mortality in monochorionic twins with selective intrauterine growth restriction. Ultrasound Obstet Gynecol, 37: 22-26. DOI: $10.1002 /$ uog. 8846

Fuente de financiamiento: Fetal Medicine Foundation Conflicto de interés: Ninguno

Correspondencia:

Enrique Gil Guevara

enrique.gil@doctors.org.uk 